\title{
Enhanced Median Flow Tracker for Videos with Illumination Variation Based on Photometric Correction
}

\author{
Asha Narayana and Narasimhadhan Venkata \\ Department of Electronics and Communication Engineering, National Institute of Technology Karnataka, India
}

\begin{abstract}
Object tracking is a fundamental task in video surveillance, human-computer interaction and activity analysis. One of the common challenges in visual object tracking is illumination variation. A large number of methods for tracking have been proposed over the recent years, and median flow tracker is one of them which can handle various challenges. Median flow tracker is designed to track an object using Lucas-Kanade optical flow method which is sensitive to illumination variation, hence fails when sudden illumination changes occur between the frames. In this paper, we propose an enhanced median flow tracker to achieve an illumination invariance to abruptly varying lighting conditions. In this approach, illumination variation is compensated by modifying the Discrete Cosine Transform (DCT) coefficients of an image in the logarithmic domain. The illumination variations are mainly reflected in the low-frequency coefficients of an image. Therefore, a fixed number of DCT coefficients are ignored. Moreover, the Discrete Cosine (DC) coefficient is maintained almost constant all through the video based on entropy difference to minimize the sudden variations of lighting impacts. In addition, each video frame is enhanced by employing pixel transformation technique that improves the contrast of dull images based on probability distribution of pixels. The proposed scheme can effectively handle the gradual and abrupt changes in the illumination of the object. The experiments are conducted on fast-changing illumination videos, and results show that the proposed method improves median flow tracker with outperforming accuracy compared to the state-of-the-art trackers.
\end{abstract}

Keywords: Illumination variation, median flow tracker, entropy, gamma correction.

Received April 6, 2017; accepted April 25, 2018

https://doi.org/10.34028/iajit/17/2/15

\section{Introduction}

Video tracking is one of the essential task used in computer vision areas such as robotics, surveillance, activity analysis, and human-computer interaction. The goal of an efficient tracker is to track an object selected in the first frame till the end frame, irrespective of changes in illumination, scale, orientation, occlusion, etc., Therefore, designing a tracking algorithm to handle the various challenges and to meet human precision is a laborious undertaking. Most of the trackers in literature are intended to address the slowly varying lighting conditions. However, they tend to fail during unpredicted lighting circumstances. Hence, in this paper, we propose to solve tracking drift of the Median Flow Tracker (MFT) [13] due to sudden changes of light in a video.

Tracking algorithms are mainly classified as generative and discriminative techniques based on appearance model. In the generative type of trackers, the object model is created using feature space and object is searched to match with the model. In this approach, template matching is performed based on pixel values [3], histogram matching [5], optical flow method [13], subspace techniques like principal component analysis [19], sparse representations [15], spatial histogram technique $[7,20]$ or particle filters [1, 12]. In contrast, discriminative type of trackers tries to separate the foreground from the background by learning through sufficient training samples. The most popular discriminative trackers use features to train the classifiers. Such methods include Haar-wavelet, oriented histograms, and local binary pattern features using Ada Boost classifier [8], Haar-like features using naive Bayes classifier [25], color statistic model using Bayes classifier [2], 2-bit pattern with random ferns classifier [14] to separate the object from its surroundings based on classification score. Recently, correlation filters have been employed to locate the target in every frame using the fast fourier transform for training and testing process $[9,10]$.

In this paper, we propose a modification of popular median flow tracker [13], which uses Lucas-Kanade (LK) point tracking method with Normalized CrossCorrelation (NCC) and Forward-Backward (FB) error measure to choose the best points in order to predict the motion of object. Since tracking is based on determining the pixel movement from the current frame to next frame, it often fails when there is a sudden change in pixel values. Hence, a novel preprocessing technique is proposed to nullify the effects of lighting conditions in this approach. Several 
algorithms exist in literature to normalize the illumination for face recognition. They include homomorphic filtering, self-quotient image [22], Discrete Cosine Transform (DCT) based normalization [4], wavelet-based normalization [6], gradient faces [26], and Weber faces [21]. The objective of these algorithms is to normalize the illumination effects of faces, which are affected by lighting conditions.

The contribution of this paper is as follows:

1. The illumination of a video is maintained constant by modifying the low-frequency coefficients of DCT of each frame in logarithmic domain based on entropy difference.

2. In addition, enhancement of each frame in a video is performed to increase the overall contrast of dull images.

3. Later, median flow tracker is applied to track an object irrespective of sudden changes in the illumination.

4. We conducted experiments using three challenging videos having sudden illumination changes and compared with the existing recent trackers in terms of precision score and overlap score.

The organization of the paper is as follows. Section 2 provides a brief review of median flow tracker. Section 3 explains the proposed illumination normalization technique for a given video. Section 4 presents video enhancement technique adopted in the proposed method. Section 5 discusses the experimental details and followed by conclusion in section 6 .

\section{Median Flow Tracker}

Median flow tracker has been proposed [13] to track an object based on point tracking with NCC and FB error measure. A block diagram of median flow tracker is depicted in Figure 1, and is explained briefly in this section to understand the working principle.

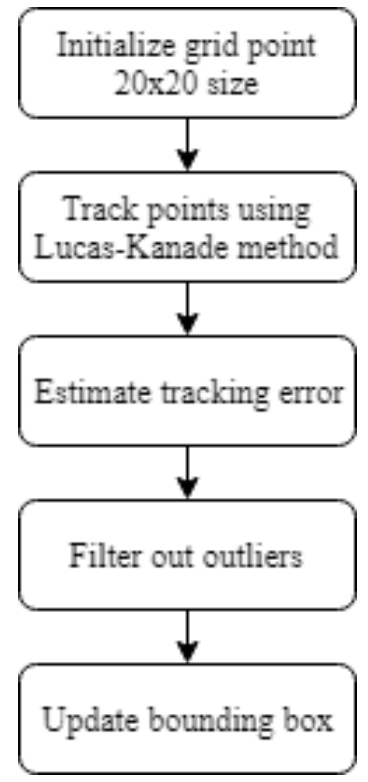

Figure 1. Block diagram of median flow tracker [12].
The tracker accepts bounding box around the object and a pair of image frames. Let $I_{f}$ and $I_{f+1}$ denote a pair of image frames, let $B_{f}$ indicates bounding box around the object in the frame $I_{f}$. Let $B_{f+1}$ denotes the bounding box obtained as the output of median flow tracker. A set of 20x20 points are initialized inside the bounding box. Each point is tracked using LK optical flow tracker to estimate the motion of an object from frame $I_{f}$ to $I_{f+l}$. Each point is associated with an error that includes NCC and Forward-Backward error (FB). NCC error is calculated based on pixel differences, whereas FB error is obtained based on Euclidean distance between forward and backward trajectories. LK tracker is utilized to estimate the forward and backward trajectories, which defines the set of tracked points from frames $f$ to $f+k$ and $f+k$ to $f$ respectively. The motion of an object is predicted by using the best points by removing the outlier points based on error. In order to estimate the scale, ratio of point distance in the present frame and that of the previous frame is calculated. The median of scale change over the set of points is used to predict the scale change in every frame. Thus, the median flow tracker achieves greater tracking results in several challenging conditions. However, the accuracy of tracker reduces for sudden illumination variation and occlusion. In this paper, we address the sudden illumination change issue in detail.

\section{Illumination Normalization}

The key objective of the proposed method is to employ illumination normalization technique as a preprocessing step. Since baseline median flow tracker is sensitive to lighting changes, we intend to modify DCT coefficients [4] to maintain the constant illumination all through the video.

Let an image $I_{f}(x, y)$ denotes the frame $f$ of a video and is composed of reflectance and illumination components [17]. i.e.,

$$
I_{f}(x, y)=I_{f}^{r}(x, y) I_{f}^{i}(x, y)
$$

Where, $I_{f}^{r}(x, y)$ represents the reflectance component which depends on object characteristics and $\mathrm{I}_{f}^{i}(x, y)$ denotes the illumination component that depends on external lighting source. The main reason for illumination variation in a video is due to changes in light intensity, which alters the pixel values. The reflectance component is constant for an object, but illumination component varies based on light concentration. Applying logarithm transform to Equation (1),

$$
\ln \quad I_{f}(x, y)=\ln \mathrm{I}_{f}^{r}(x, y)+\ln \mathrm{I}_{f}^{i}(x, y)
$$

Consider the next frame $I_{f+l}(x, y)$ with different illumination component compared to the present frame $I_{f}(x, y)$. Since the object property remains same and, 
only illumination component $\varepsilon$ is added or subtracted from $I_{f}(x, y)$ as given by,

$$
\begin{gathered}
\ln \mathrm{I}_{f+1}(x, y)=\ln \mathrm{I}_{f}(x, y) \pm \varepsilon \\
\ln \mathrm{I}_{f+1}(x, y)=\ln \mathrm{I}_{f+1}^{r}(x, y)+\ln \mathrm{I}_{f+1}^{i}(x, y)
\end{gathered}
$$

Since $\ln I_{f}^{r}(x, y)=\ln I_{f+1}^{r}(x, y)$, hence $\varepsilon \quad$ is obtained as, $\varepsilon=\ln I_{f+1}^{i}(x, y)-\ln I_{f}^{i}(x, y)$.

Therefore, the difference between two images is the desired change in illumination which needs to be corrected to maintain the constant intensity over a video. The discrete cosine transform has been used in signal processing area due to its high energy compaction property. Illumination variations can be well compensated by adding or subtracting the difference $\varepsilon$ in the logarithmic domain. Since illumination is a slowly varying component in an image than the reflectance component, hence lowfrequency coefficients in the DCT domain are related to the illumination component. DC coefficient of DCT domain determines the overall illumination of an image. Therefore, the desired uniform illumination can be obtained by setting the DC coefficient to a constant value in every frame. In order to get uniform illumination during the video, running average of frames is accomplished with a learning rate of $\lambda=0.01$. However, very low and highly illuminated frames are excluded from an average calculation based on entropy difference. The entropy of an image is obtained as

$$
E=-\sum_{k} p(k) \log _{2} p(k)
$$

Where $p(k)$ denotes the probability density function of image pixels. Hence, the average image at the current frame is determined only if the entropy difference between the present and previous frame differs by a threshold of $\theta$. i.e.,

$$
|E(\mathrm{f})-E(\mathrm{f}-1)|<\theta
$$

Where $f$ denotes the frame number. In the proposed scheme, $\theta$ is set at 5 .

Therefore, an average image is calculated using,

$$
s(x, \mathrm{y})=\mathrm{s}(\mathrm{x}, \mathrm{y})+\lambda \mathrm{I}_{f}(x, y)
$$

Mean of average image model is computed as,

$$
\text { meanS }=\frac{1}{M \times N} \sum_{x} \sum_{y} \ln \mathrm{s}(x, y)
$$

Thus, the desired uniform illumination can be obtained by setting the DC coefficient of every frame to mean $S \times \sqrt{M \times N}$, where $(\mathrm{M}, \mathrm{N})$ denotes the size of each frame. As mentioned before, low-frequency DCT coefficients are highly related to illumination variations and should be discarded to compensate for illumination changes. Thus upper left corner of DCT image represents low-frequency factors which are set to zero by arranging the coefficients in a zig-zag manner as shown in Figure 2. In the proposed approach, 25 coefficients are set to zero. Also, DC coefficient in DCT domain is replaced by mean $S \times \sqrt{M \times N}$, where meanS given by Equation (8). Henceforth, inverse DCT is applied to get normalized image frame with constant intensity throughout the video. The entropy of DCT normalized image remains almost uniform compared to the original image as depicted in Figures 3 and 4 details about how illumination effects are nullified using the proposed method.

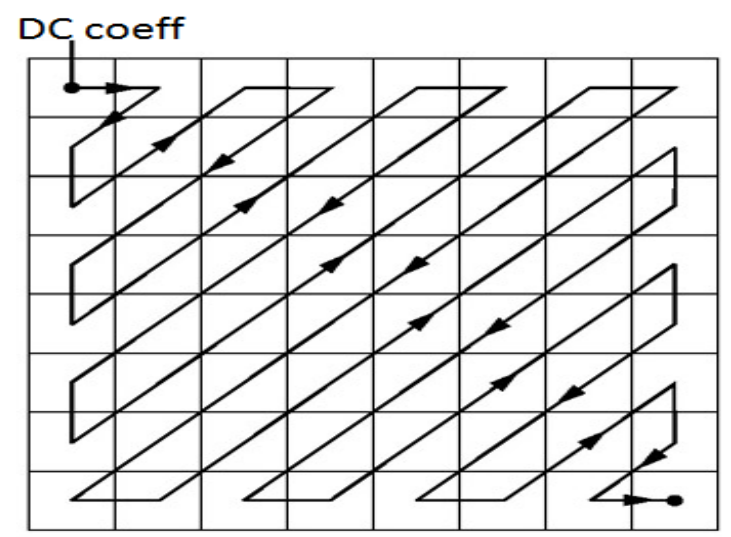

Figure 2. Zig-zag order of DCT coefficients.

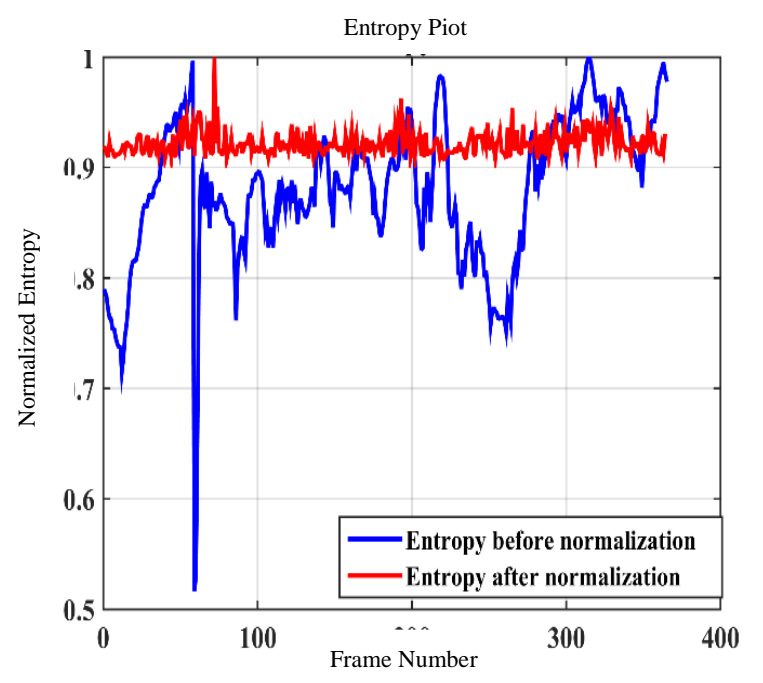

Figure 3. The entropy modification using DCT based illumination normalization technique. 


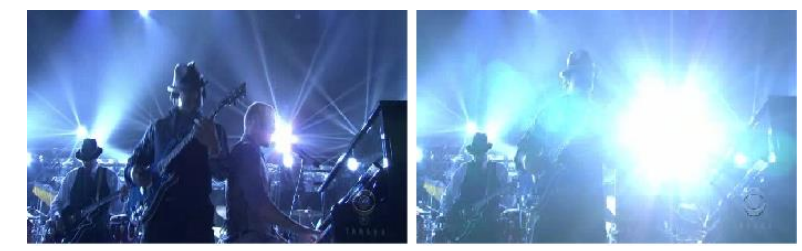

a) Original image at frame number 59. b) Original image at frame number 60 .
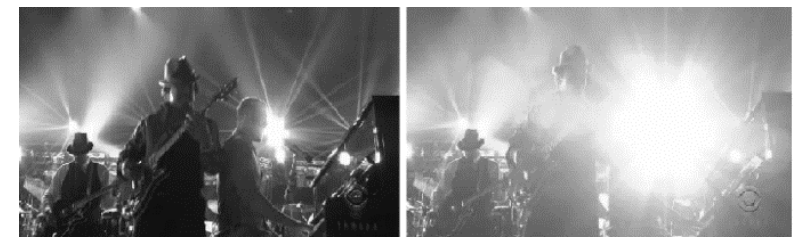

c) Gray scale image at frame number 59. d) Frame number at frame number 60 .

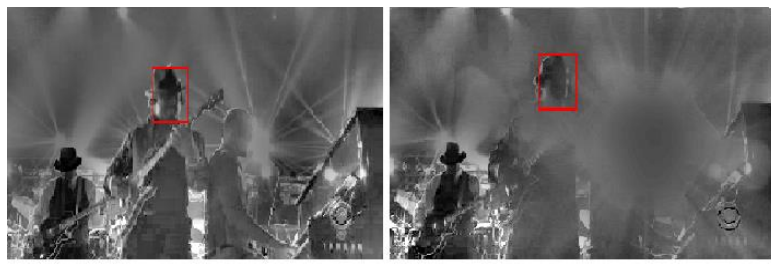

e) Illumination normalized image using f) Illumination normalized image using DCT at frame number 59.

DCT at frame number 60.

Figure 4. Illustration of the proposed illumination invariant technique on 2 frames of shaking video.

\section{Video Enhancement}

After normalizing illumination of each frame, enhancement technique is applied based on algorithm proposed in [11]. Let $x$ denotes the pixel value of an image. The adaptive gamma correction uses $\operatorname{cdf}(x)$ as the adaptive parameter for gamma correction method. Point transformation on pixel value $x$ is defined as

$$
T(x)=x_{\max }\left(\frac{x}{x_{\max }}\right)^{\gamma}
$$

Where $x_{\max }$ denotes maximum value of $x$. Weighting distribution function is computed to modify the histogram of an image as follows:

$$
p d f(x)=p d f_{\max }\left(\frac{p d f(x)-p d f_{\min }}{p d f_{\max }-p d f_{\min }}\right)^{\alpha}
$$

Where $a$ is the adjusted parameter and set to 0.6 in the proposed tracking method. $p d f(x)$ represents the histogram of an image with a maximum of $p d f_{\max }$ and a minimum of $p d f_{\min }$. Cumulative density function $c d f(x)$ is obtained as given below,

$$
c d f(x)=\sum_{x=0}^{255} \frac{p d f(x)}{p d f_{\text {sum }}}
$$

Where $p d f_{\text {sum }}=\sum_{x=0}^{255} p d f(x)$.

Adaptive parameter gamma $y$ is calculated based on $c d f(x)$ to enhance the video in every frame using the Equation (9) and is given by

$$
\gamma=1-c d f(x)
$$

The image contrast enhancement helps to improve tracking accuracy. The proposed algorithm for tracking using DCT based illumination normalization, gamma enhancement, and median flow tracker is summarized in Algorithm 1.

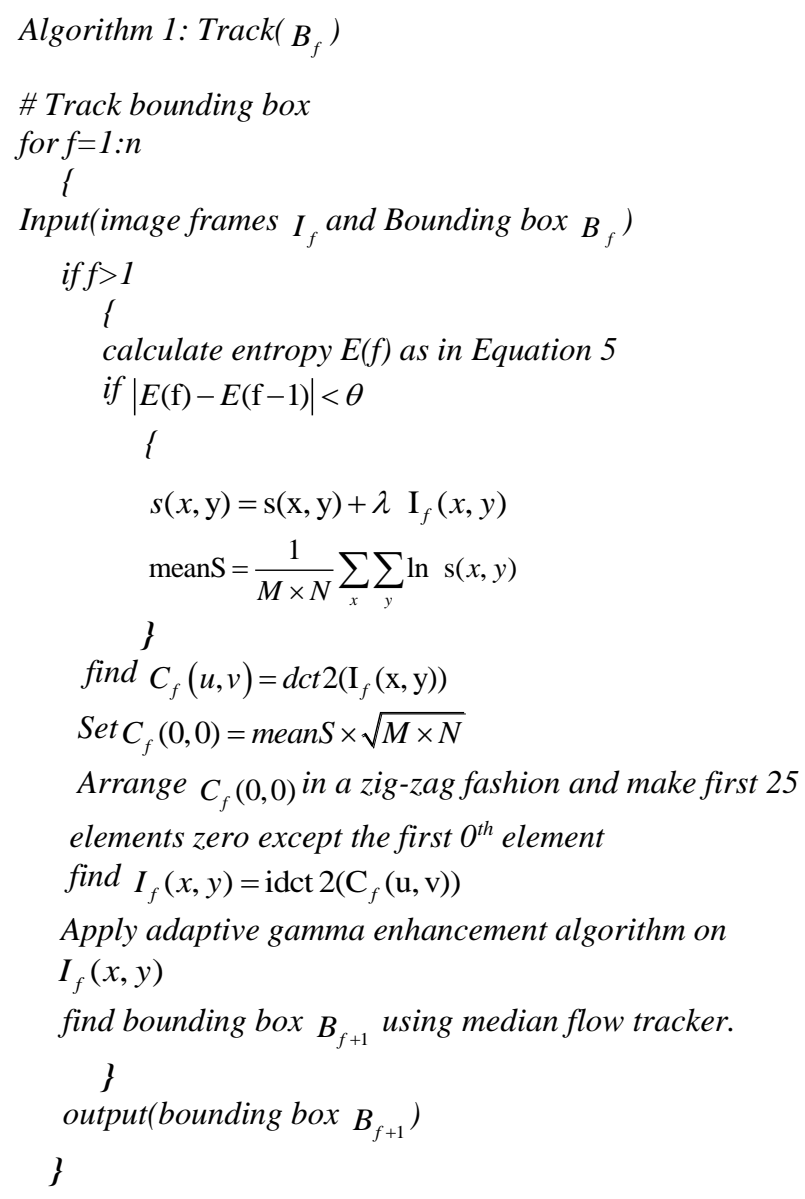

\section{Experimental Setup}

The proposed algorithm is implemented using MATLAB 15a software in a machine with Intel(R) Core i5-5200U, CPU at the $2.20 \mathrm{GHz}$ processor with 8GB RAM. The proposed tracking method is evaluated on three image sequences selected from Object Tracking Benchmark (OTB) dataset [23] which has sudden illumination change as a challenging aspect.

In this section, we provide the details of datasets, quantitative and qualitative analysis to demonstrate the effectiveness of the proposed tracker. Also, we compare the proposed tracker with baseline tracker, i.e., median flow tracker [12] and tracking, learning, and detection [13] separately.

\subsection{Dataset}

The proposed tracker is implemented and evaluated on three challenging videos. The enhanced median flow tracker is designed to solve sudden changes in illumination, and it does not affect the performance of baseline tracker in other challenging aspects. Hence, we have selected the videos from OTB dataset [23] which pose sudden illumination change at many places. The videos are shaking, singer 2 and man. 


\subsection{Quantitative Performance Evaluation}

The proposed tracker is named as illumination Invariant Median Flow Tracker (IVMFT) and is compared with the existing tracking methods which are very recent and more popular in video tracking field such as Corrected Background-Weighted Histogram (CBWH) [16], Distractor-Aware Tracker (DAT) [18], Compressive Tracking (CT) [25], Incremental Discriminative Color Object Tracking (IDCT) [2], Enhanced Distribution Field Tracking (EDFT) [7], Tracking, Learning and Detection (TLD) [14], Distribution Field Tracking (DFT) [20], Circulant Structure Kernel Based Tracking (CSK0 [9].

Two quantitative measures are used to compare the proposed tracker with the existing trackers which include Distance Precision score (DP) and Overlap Precision score (OP) [23]. In order to find the deviation of a tracker, centre location error is employed, which is calculated as the Euclidean distance between ground-truth and tracked locations. Distance precision score determines the percentage of frames with the centre location error less than the threshold of 20 pixels. Overlap precision score is utilized to evaluate a tracker, based on overlap condition. Hence, bounding box overlap is calculated from tracked area and ground-truth area as follows:

$$
A=\frac{\left|B_{t} \cap G_{t}\right|}{\left|B_{t} \cup G_{t}\right|},
$$

where $B_{t}$ represents tracked area, $G_{t}$ denotes groundtruth area, $\cap$ represents the intersection operator, $\cup$ denotes the union operator and || denotes the area bounded by a region. To measure the overlap precision score of a tracker, percentage of a number of successful frames whose overlap $A$ larger than the threshold of 0.5 is used. Precision plots and success plots show distance precision score and overlap precision score for various range of thresholds respectively. From the experimental analysis, IVMFT performs best compared to existing trackers in terms of distance precision score and overlap precision score as shown in Tables 1,2, and 3. A comparative analysis of enhanced Median Flow Tracker (MFT) and tracking, learning and detection (TLD) is tabulated in Table 1 and depicted in Figures 5 and 6. Furthermore, precision plots and success plots of state-of-the-art trackers are depicted in Figures 7 and 8.

Table 1. Comparison between MFT, improved median flow tracker IVMFT and tracker TLD.

\begin{tabular}{|c|c|c|c|c|c|c|c|c|c|}
\hline & \multicolumn{3}{|c|}{ MFT } & \multicolumn{3}{c|}{ IVMFT } & \multicolumn{3}{c|}{ TLD } \\
\cline { 2 - 10 } & Shaking & Singer2 & Man & Shaking & Singer2 & Man & Shaking & Singer2 & Man \\
\hline $\begin{array}{c}\text { Distance } \\
\text { Precision }\end{array}$ & $\mathbf{1 5 . 6 2}$ & $\mathbf{1 0 . 9 3}$ & 100 & 100 & 21.58 & 100 & 43.39 & 13.66 & 100 \\
\hline $\begin{array}{c}\text { Overlap } \\
\text { Precision }\end{array}$ & 15.62 & $\mathbf{1 0 . 9 3}$ & 36.57 & 82.47 & 20.22 & 100 & 39.45 & 13.39 & 49.45 \\
\hline
\end{tabular}

Table 2. Distance precision score of state-of-the-art trackers for three sequences that address sudden illumination changes as a challenge.

\begin{tabular}{|c|c|c|c|c|c|c|c|c|c|c|}
\hline & CBWH & CSK & CT & DAT & DFT & EDFT & IDCT & TLD & IVMFT & MFT \\
\hline shaking & 2.74 & 76.99 & 4.11 & 3.29 & 83.01 & 16.71 & 1.37 & 43.29 & 100.00 & 15.62 \\
\hline singer2 & 0.27 & 4.10 & 0.82 & 1.64 & 62.84 & 56.01 & 28.69 & 13.66 & 21.58 & 10.93 \\
\hline man & 29.10 & 100.00 & 22.39 & 100.00 & 23.13 & 23.13 & 25.37 & 100.00 & 100.00 & 100.00 \\
\hline Mean & 10.71 & 60.36 & 9.11 & 34.98 & 56.33 & 31.95 & 18.48 & 52.32 & 73.86 & 42.18 \\
\hline
\end{tabular}

Table 3. Overlap precision score of state-of-the-art trackers for three sequences that address sudden illumination changes as a challenge.

\begin{tabular}{|c|c|c|c|c|c|c|c|c|c|c|}
\hline & CBWH & CSK & CT & DAT & DFT & EDFT & IDCT & TLD & IVMFT & MFT \\
\hline shaking & 1.64 & 58.08 & 3.01 & 3.01 & 82.47 & 16.16 & 1.10 & 39.45 & 82.47 & 15.62 \\
\hline singer2 & 0.27 & 3.55 & 1.37 & 1.37 & 69.67 & 59.56 & 25.96 & 13.39 & 20.22 & 10.93 \\
\hline man & 20.90 & 100.00 & 0.75 & 47.01 & 22.39 & 22.39 & 23.13 & 95.52 & 100.00 & 36.57 \\
\hline Mean & 7.60 & 53.88 & 1.71 & 17.13 & 58.18 & 32.71 & 16.73 & 49.45 & 67.56 & 21.04 \\
\hline
\end{tabular}

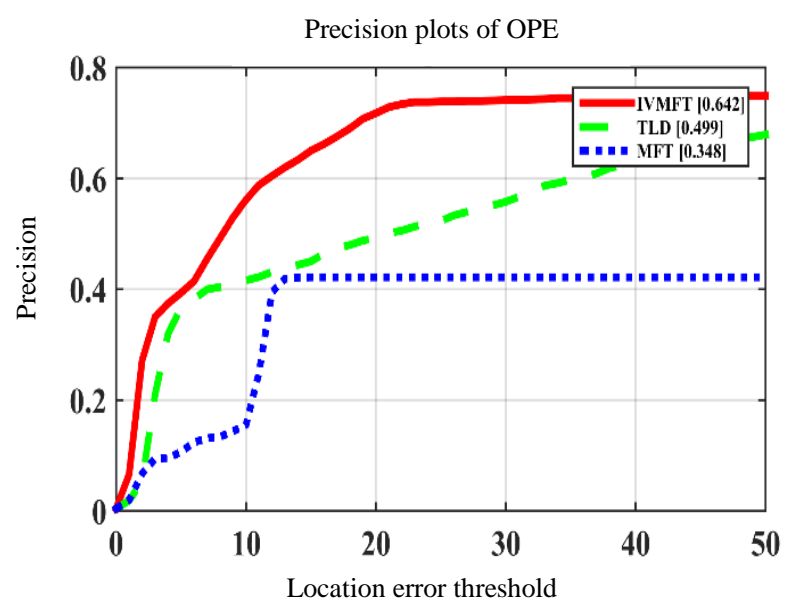

Figure 5. The comparison of precision plots of IVMFT (enhanced MFT), MFT and TLD.

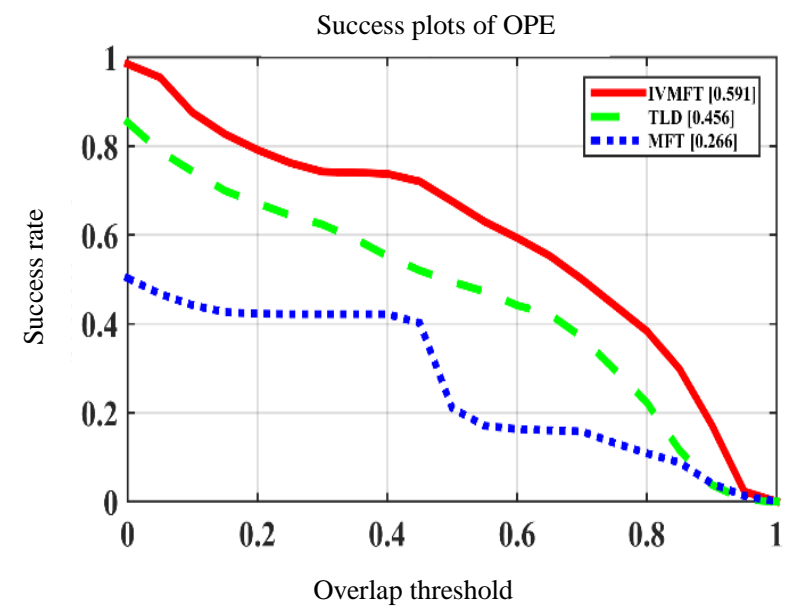

Figure 6. The comparison of precision plots of IVMFT (enhanced MFT), MFT and TLD. 


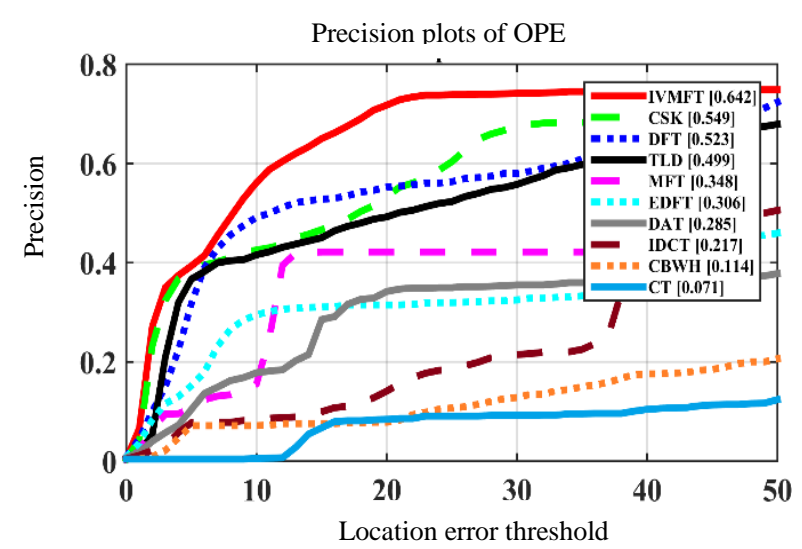

Figure 7. Precision plots of the state-of-the-art trackers for 3 image sequences with sudden illumination change as a challenging aspect.

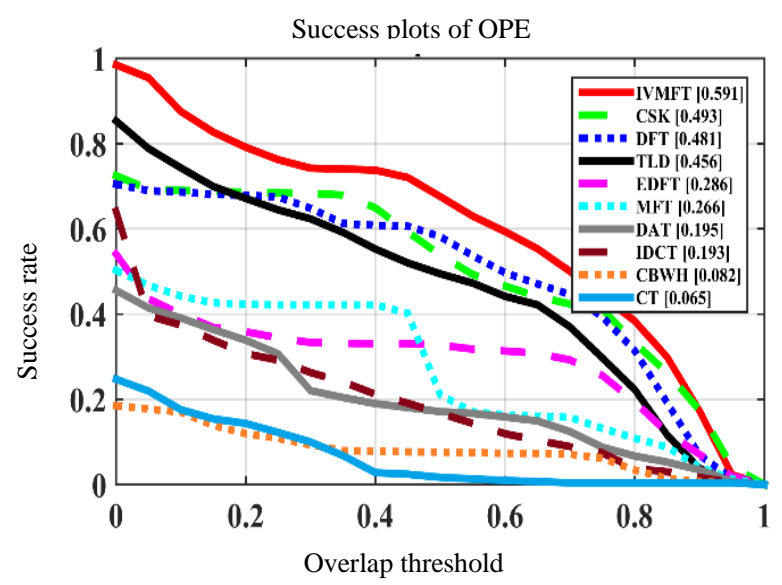

Figure 8. Success plots of the state-of-the-art trackers for 3 image sequences with sudden illumination change as a challenging aspect.

\subsection{Qualitative Performance Evaluation}

Figure 9 depicts shaking video, which shows a person (target) singing in a music program with flash light effects. It can be observed that the light changes are very sudden. Since, the base tracker is designed using optical flow technique which is sensitive to light changes. Hence, modified tracker IVMFT is intended to nullify the illumination effect and tracks a person in shaking sequence till video completion. Flash light effects are observed in many frames like $60^{\text {th }}$, where median flow tracker fails to follow an object, however, the proposed tracker is very robust in handling such cases. Also, CSK tracker is able to track the complete sequence, whereas rest of the trackers fail due to illumination changes.

In Figure 10 man video sequence is presented, in which a person moves in a dark room and is suddenly illuminated. Hence, there is a large variation of light and IVMFT is successful in tracking the complete sequence. MFT deviates from tracking object due to the sudden change in light, whereas the proposed tracker is made illumination independent to track all the way through the sequence. However, recent trackers like DFT, EDFT, CBWH fail to track the complete sequence.
In Figure 11. Singer 2 video frames are presented, which is having more backlight effects due to which many trackers cannot track. However, proposed tracker do not miss the target due to an unexpected change in illumination, however fails due to heavy pose variation.
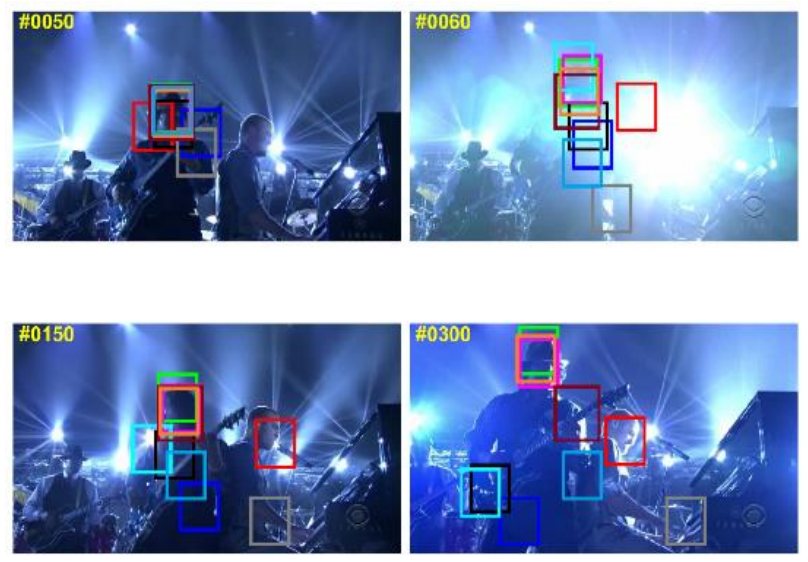

Figure 9. Qualitative evaluation of IVMFT with state-of-the-art tracker for shaking sequence.
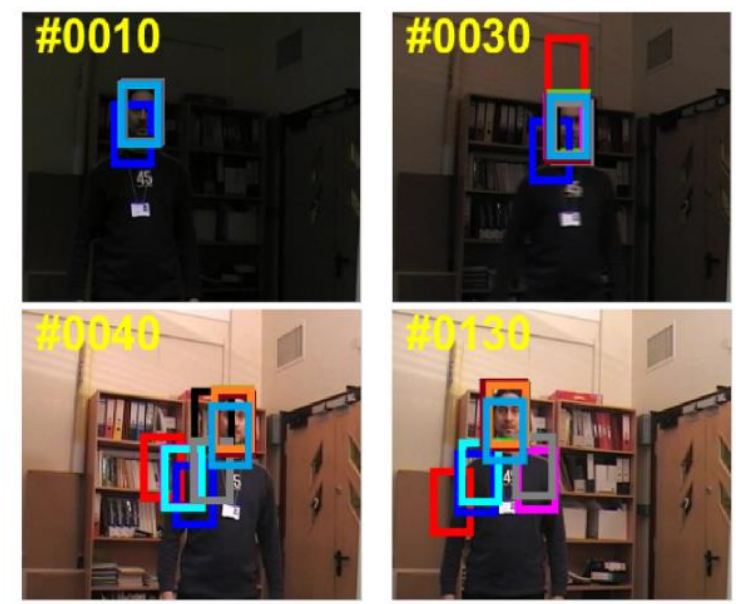

Figure 10. Qualitative evaluation of IVMFT with state-of-the-art tracker for man sequence.
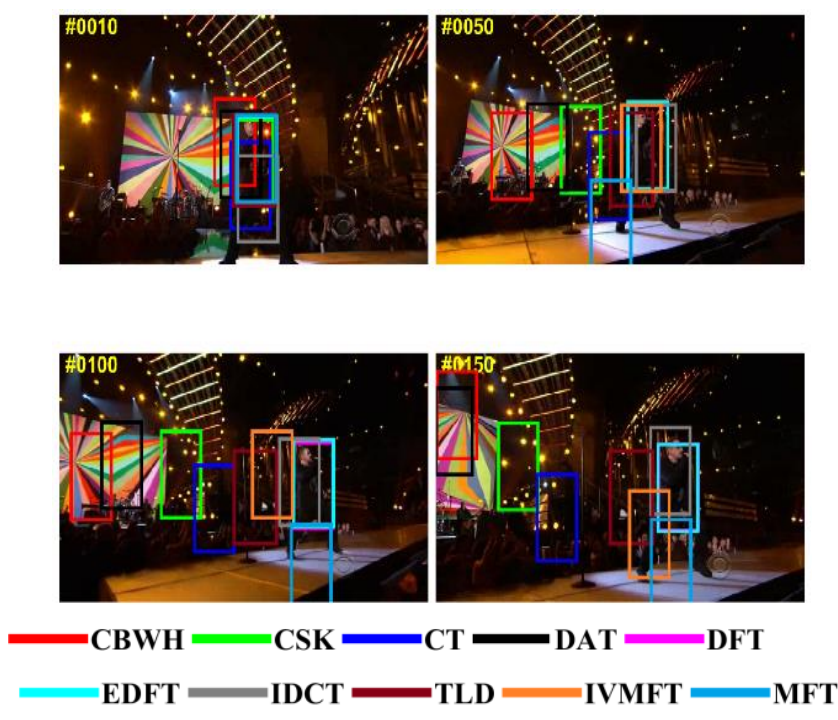

Figure 11. Qualitative evaluation of IVMFT with state-of-the-art trackers for singer2 sequence. 


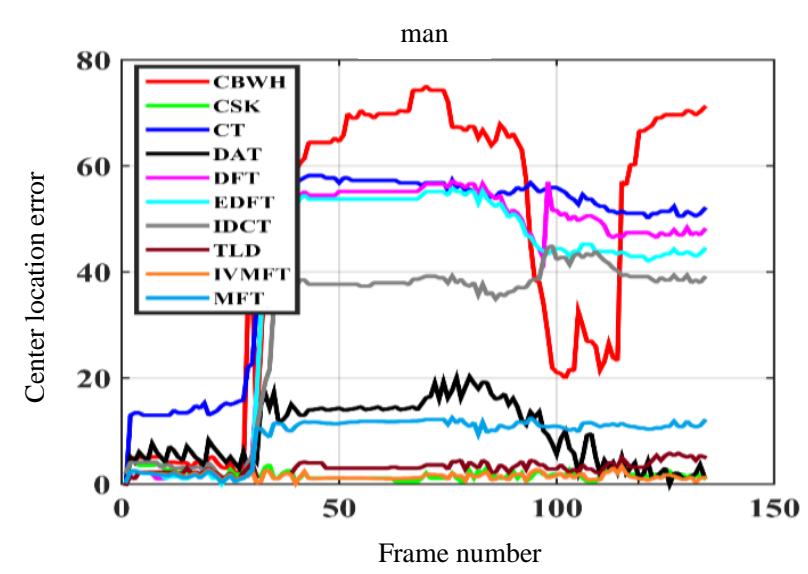

Figure 12. Centre location error graph of man video sequences.

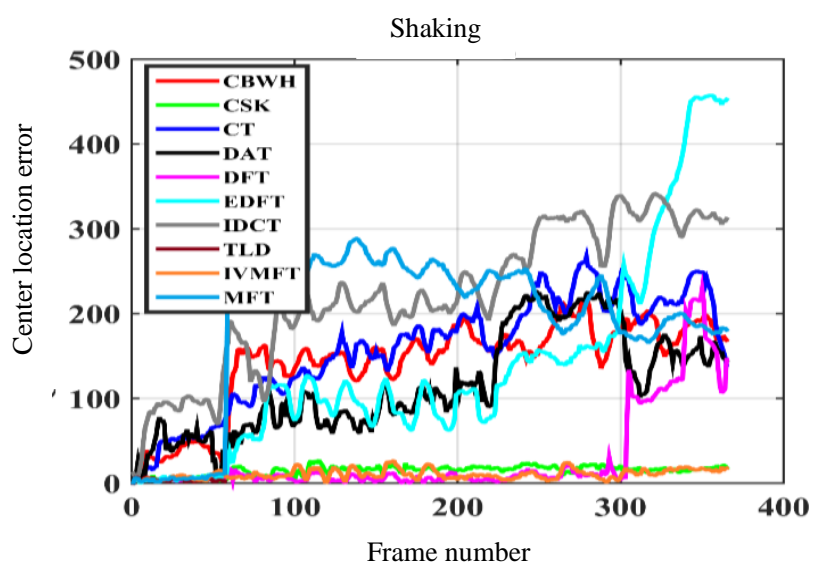

Figure 13. Centre location error graph of shaking video sequences.

Figures 12 and 13 depict centre location error graph of all the trackers for man and shaking video sequences respectively; the proposed tracker shows the least error as compared with the existing trackers.

\section{Conclusions}

In this paper, we proposed a simple but an efficient framework to track an object under abrupt varying illumination environment within and between frames. To undertake this issue, DCT coefficients are tailored to eliminate the effect of illumination changes. In addition, the contrast of an image is improved using an adaptive gamma correction technique. Several video sequences with sudden changing illumination have been considered to test the proposed tracker. The experimental results demonstrated that the proposed algorithm improves the median flow tracker for slow/abrupt changes in the illumination. In addition, the proposed technique can also be useful for various tracker which is sensitive to unexpected lighting changes. However, the proposed method is not robust against occlusion, which could be achieved by embedding re-detection unit as in TLD tracker.

\section{References}

[1] Abdennour S. and Tebbikh H., "Parallel Particle Filters for Multiple Target Tracking," The
International Arab Journal of Information Technology, vol. 13, no. 6, pp. 707-714, 2016.

[2] Asvadi A., Mahdavinataj H., Karami M., and Baleghi Y., "Incremental Discriminative Color Object Tracking," in Proceedings of International Symposium on Artificial Intelligence and Signal Processing, Tehran, pp. 71-81, 2013.

[3] Briechle K. and Hanebeck U., "Template Matching Using Fast Normalized Cross Correlation," in Proceedings of The International Society for Optical Engineering Control, Orlando, pp. 95-102, 2001.

[4] Chen W., Er M., and Wu S., "Illumination Compensation and Normalization for Robust Face Recognition Using Discrete Cosine Transform in Logarithm Domain," IEEE Transactions on Systems, Man, and Cybernetics, Part B (Cybernetics), vol. 36 , no. 2, pp. 458466, 2006.

[5] Comaniciu D., Ramesh V., and Meer P., "RealTime Tracking of Non-Rigid Objects Using Mean Shift," in Proceedings IEEE Conference on Computer Vision and Pattern Recognition, Hilton Head Island, pp. 142-149, 2000.

[6] Du S. and Ward R., "Wavelet-Based Illumination Normalization For Face Recognition," in Proceedings of IEEE International Conference on Image Processing, Genova, pp. II-954, 2005.

[7] Felsberg M., "Enhanced Distribution Field Tracking Using Channel Representations," in Proceedings of the IEEE International Conference on Computer Vision Workshops, Sydney, pp. 121-128, 2013.

[8] Grabner H., Grabner M., and Bischof H., "RealTime Tracking Via On-Line Boosting," in Proceedings of British Machine Vision Conference, Edinburgh, pp. 47-56, 2006.

[9] Henriques J., Caseiro R., Martins P., and Batista J., "Exploiting the Circulant Structure of Tracking-By-Detection with Kernels," in Proceedings of European Conference on Computer Vision, Florence, pp. 702-715, 2012.

[10] Henriques J., Caseiro R., Martins P., and Batista J., "High-Speed Tracking with Kernelized Correlation Filters," IEEE Transactions on Pattern Analysis and Machine Intelligence, vol. 37, no. 3, pp. 583-596, 2015.

[11] Huang S., Cheng F., and Chiu Y., "Efficient Contrast Enhancement Using Adaptive Gamma Correction with Weighting Distribution," IEEE Transactions on Image Processing, vol. 22, no. 3, pp. 1032-1041, 2013.

[12] Islam Z., Oh C., and Lee C., "Effect of Resampling Steepness on Particle Filtering Performance in Visual Tracking," The International Arab Journal of Information Technology, vol. 10, no. 1, pp. 102-109, 2013. 
[13] Kalal Z., Mikolajczyk K., and Matas J., "Forward-Backward Error: Automatic Detection of Tracking Failures," in Proceedings of International Conference on Pattern Recognition, Istanbul, pp. 2756-2759, 2010.

[14] Kalal Z., Mikolajczyk K., and Matas J., "Tracking Learning Detection," IEEE Transactions on Pattern Analysis and Machine Intelligence, vol. 34, no. 7, pp. 1409-1422, 2012.

[15] Nhat V. and Lee G., "Illumination Invariant Object Tracking with Adaptive Sparse Representation," International Journal of Control, Automation and Systems, vol. 12, no. 1, pp. 195-201, 2014.

[16] Ning J., Zhang L., Zhang D., and Wu C., "Robust Mean-Shift Tracking with Corrected Background-Weighted Histogram," IET Computer Vision, vol. 6, no. 1, pp. 62-69, 2012.

[17] Phadke G. and Velmurugan R., "Improved Weighted Histogram for Illumination Invariant Mean-Shift Tracking," in Proceedings of the Indian Conference on Computer Vision Graphics and Image Processing, Bangalore, pp. 1-8, 2014.

[18] Possegger H., Mauthner T., and Bischof H., "In Defense of Color-Based Model-Free Tracking," in Proceedings of IEEE Conference on Computer Vision and Pattern Recognition, Boston, pp. 2113-2120, 2015.

[19] Ross D., Lim J., Lin R., and Yang M., "Incremental Learning for Robust Visual Tracking," International Journal of Computer Vision, vol. 77, no. 1, pp. 125-141, 2008.

[20] Sevilla-Lara L. and Learned-Miller E., "Distribution Fields for Tracking," in Proceedings of IEEE Conference on Computer Vision and Pattern Recognition, pp. 1910-1917, 2012.

[21] Wang B., Li W., Yang W., and Liao Q., "Illumination Normalization Based on Weber's Law with Application to Face Recognition," IEEE Signal Processing Letters, vol. 18, no. 8, pp. 462-465, 2011.

[22] Wang H., Li S., Wang Y., and Zhang J., "Self Quotient Image for Face Recognition," in Proceedings of International Conference on Image Processing, Singapore, pp. 1397-1400, 2004.

[23] Wu Y., Lim J., and Yang M., "Online Object Tracking: A Benchmark," in Proceedings of the IEEE Conference on Computer Vision and Pattern Recognition, Portland, pp. 2411-2418, 2013.

[24] Zhang K., Zhang L., and Yang M., "Fast Compressive Tracking," IEEE Transactions on Pattern Analysis and Machine Intelligence, vol. 36, no. 10, pp. 2002-2015, 2014.

[25] Zhang K., Zhang L., and Yang M., "Real-Time Compressive Tracking," in Proceedings of
European Conference on Computer Vision, Florence, pp. 864-877, 2012.

[26] Zhang T., Tang Y., Fang B., Shang Z., and Liu $X$. "Face Recognition Under Varying Illumination Using Gradientfaces," IEEE Transactions on Image Processing, vol. 18, no. 11, pp. 2599-2606, 2009.

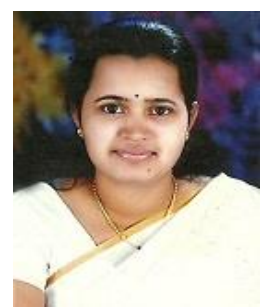

Asha Narayana received B E and $\mathrm{M}$ Tech degree in Electronics and Communication Engineering from NMAMIT Nitte in 2006 and 2012 respectively. She is currently pursuing Ph.D. in National Institute of Technology Karnataka, India (NITK). Her research interests include computer vision and medical image processing.

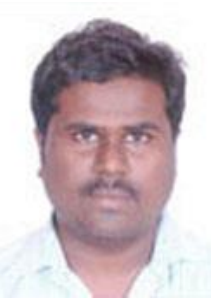

Narasimdhan Venkata received $\mathrm{BE}$ degree in Electronics and Communication Engineering from Andhra University, in 2005 and M Tech degree in Signal Processing from Indian Institute of Technology, Guwahati, India, in 2007. Later, He received his Ph.D. degree from Indian Institute of Science, India, in 2012. He is presently working as an Assistant Professor in department of Electronics and Communication Engineering at National Institute of Technology, Karnataka, India. His research interests include medical imaging, computer vision, and medical image processing 